\title{
The delicate balance of patient autonomy and compassionate care
}

\author{
Rodney H. Breau, MSc, MD, FRCSC \\ Division of Urology, Department of Surgery, The Ottawa Hospital/University of Ottawa, Ottawa, ON, Canada
}

Cite as: Breau RH. The delicate balance of patient autonomy and compassionate care. Can Urol Assoc J 2021;15(4):114. http://dx.doi.org/10.5489/cuaj.7251

See related paper on page 108

"I'm calling for my test results. Negative? Oh, my God. WHY! WHY! WHY? What? What? Negative is good? Oh, yes of course! How stupid of me. Thank you."

- George Costanza, Seinfeld

M any of us believe that the medical record belongs to the patient. For years, we have printed and provided radiology and pathology reports for interested patients. We explained the medical jargon, and many patients left the encounter feeling informed and empowered.

Providing these documents to most patients, without context, seems unwise. The majority of patients do not have the medical literacy to understand the reports, which can lead to unwarranted anxiety. A more concerning scenario is one in which the patient understands a report that reveals upsetting information about his/her health. As physicians, one of our most sacred responsibilities is informing patients of bad news with compassion and support.

My institution adopted a policy of real-time access to medical reports. As soon as a test result becomes available, patients receive an email link prompting them to view their results. I have no doubt this was a well-intentioned policy. However, I am confident that this method of information sharing has not improved the patient experience. All of the common-sense repercussions have come to pass.

My practice is exclusively cancer-related. We know these patients often worry about test results. Predictably, upon reading a medical report without context, many patients are highly concerned by their interpretation - sometimes due to misunderstanding, and sometimes due to an accurate interpretation. As surgeons, we are not always immediately available to support these patients in the manner they deserve. This has placed a significant burden on our administrative staff, who field these calls. I frequently receive a text or email from my assistant after a long OR day stating that a patient is highly distressed, believing they have metastases. Just as concerning is the patient who worried for days or weeks prior to their appointment. It is telling that I have never had a patient inform me that they were very happy to have early access to their medical record. In fact, I have heard the opposite.

In this issue, Perlis and colleagues report on their process to improve patient understanding of a prostate magnetic resonance imaging report. ${ }^{1}$ Through an iterative process with patients, they have developed a method for communicating results. I believe this is a valuable contribution and I hope it is adopted at hospitals across Canada, and beyond. As with all patient communication tools, we should learn about the clinical usefulness of this instrument in different languages, geographic settings, and across the spectrum of patient literacy. Most importantly, we should determine the effect of these reports on patient well-being, knowledge, and satisfaction.

I believe the authors have provided a nice framework for development of patient-centered radiology reports that can be adapted to other radiology and non-radiology test results. Hopefully, through patient-centered reports, we will be able to mitigate some anxiety related to test misinterpretation. It is still my belief that breaking bad news to patients is best done between a caring physician, patients, and their loved ones. Finding the balance between patient autonomy, access to personal information, and compassionate care is a laudable goal.

Competing interests: The author reports no competing personal or financial interests related to this work.

\section{Reference}

1. Perlis N, Finelli A, Lovas $M$, et al. Creating patient-centered radiology reports to empower patients undergoing prostate magnetic resonance imaging. Can Urol Assoc J 2021;15:108-13. http://dx.doi. org/10.5489/cuaj.6585

Correspondence: Dr. Rodney H. Breau, Division of Urology, Department of Surgery, The Ottawa Hospital/University of Ottawa, Ottawa, ON, Canada; rbreau@toh.ca 